Sharif University of Technology
Scientia Iranica
SCIENTIA
IRAN I CA

\title{
Numerical study of insulation structure characteristics and arrangement effects on cell trapping using alternative current insulating based dielectrophoresis
}

\author{
R. Javidi ${ }^{a}$, M. Moghimi Zand ${ }^{\mathrm{a}, \mathrm{b}, *}$, and R. Nouri ${ }^{\mathrm{a}}$ \\ a. Small Medical Devices, BioMEMS \& LoC Lab, School of Mechanical Engineering, College of Engineering, University of Tehran, \\ Tehran, Postal Code 14399-55961, Iran. \\ b. Department of Mechanical Engineering, College of Engineering, Michigan State University, MI, USA.
}

Received 15 March 2018; accepted 26 January 2019

\section{KEYWORDS \\ Insulator-based dielectrophoresis; Insulating structure; \\ Optimal design; \\ Trapping efficiency.}

\begin{abstract}
Insulator-based dielectrophoresis is a recently developed technique in which insulating posts are used to produce non-uniformity in the electric field in a microchannel. This study presents the effects of insulating posts' geometry and arrangement on the trapping efficiency of red blood cells in an alternating current-insulator-based dielectrophoresis system. Microchannels containing square, circular, and diamond-shaped posts with particles under the influence of positive dielectrophoresis force and fluid flow were considered in this study. The finite element method was used to compute the velocity of the flow and electric field. The numerical method was verified by comparing the numerical results with experimental data. Two distinct criteria for examining particle trapping for distinct shapes and arrangements of insulating posts were introduced. Particle tracing simulation was implemented to observe particle trapping and compare the trapping performance of systems with distinct posts. As shown in the results for the system with circular and square posts, insulators should be narrowed to improve particle trapping, while the diamond post should be widened to increase the trapping efficiency. In addition, the particle tracking results showed that the microchannel with square posts was more efficient in particle trapping.

(C) 2020 Sharif University of Technology. All rights reserved.
\end{abstract}

\section{Introduction}

Dielectrophoresis (DEP) is one of the most commonly used techniques for manipulating microparticles, cells, viruses, DNA, and other components in microfluidic devices. DEP microfluidic devices are employed for trapping, isolating, classifying, and identifying micro and nanoparticles [1-4]. Trapping and isolation of

\footnotetext{
*. Corresponding author.

E-mail address: mahdimoghimi@ut.ac.ir (M. Moghimi Zand)
}

doi: $10.24200 /$ sci.2019.50632.1794 bioparticles is an important part of many processes in biological and biomedical applications. DEP is described as the electrokinetic motion of a particle caused by polarization effects in the presence of nonuniform electric fields [5-7]. The response of particles to DEP force is dependent on the physical and electrical properties of the particle and surrounding media, particle volume, and the spatial gradient of the square of the electric field and the frequency [8-11].

In conventional techniques, an interdigitated electrode array, through which the sample fluid passes to generate Alternating Current (AC) electric fields, is employed [12-14]. This non-uniform electric field produces DEP force in the microchip. This DEP tech- 
nique is utilized in the manipulation, separation, and enrichment of bioparticles $[1,15,16]$; however, electrode fouling, bubble formation (caused by electrolysis), and delamination are the drawbacks of this method [17]. Insulator-based Dielectrophoresis (iDEP) is an alternative approach to concentrating and trapping bioparticles with embedding insulating obstacle between two electrodes [18]. In the iDEP technique, the nonuniform electric field can be produced with alternating (AC) and Direct Current (DC) potentials. In DC-iDEP, two external electrodes are placed at the reservoirs of the microchip. In microchips based on DC-iDEP, the particles are under the influence of the combination of electrophoresis, electroosmosis, and DEP [19-22]. Hernandez et al. [23] illustrated the separation of E. coli and yeast cells by introducing a DCiDEP microfluidic device that contains a microchannel with cylindrical insulating posts. In this paper, the effects of dielectric properties of different cells and media were considered to predict the behavior of cells in the microchannel.

Microchannel geometry has significant impacts on the gradient of an electric field that, in turn, influences particle trapping $[24,25]$. The authors found that the geometry of an insulating post with sharper angles required lower applied voltage to trap the particle. The effects of the insulating post geometry and material on the distortion of the electric field using the multipole expansion method were studied by Pesch et al. [26]. This paper investigated the influence of the sharpness of the post's edge on particle trapping efficiency. Lalonde et al. [27] investigated the effect of circular and diamond-shaped insulating structures on bioparticle trapping of a DC-iDEP microfluidic device. They studied the effect of various insulator posts on the gradient square of an electric field to reach high trapping efficiency. Mohammadi et al. [28] optimized circular insulating post spaces to minimize the required voltage for the particle trapping in a DC-iDEP microdevice. This optimization can be utilized for high trapping efficiency and reduce the influence of Joule heating on bioparticles.

In addition to negative DEP employed in most of the studies, positive dielectrophoresis (pDEP) can be utilized to manipulate bioparticles. pDEP systems deal with $\mathrm{AC}$ voltage depending on hydrodynamic pumps to trap bioparticles [29-32]. In these systems, particles are trapped on the surface of the insulating posts at the maximum gradient squared of electric field regions. Sano et al. [33] introduced a novel microfluidic device for manipulating bioparticles with a combination of contactless dielectrophoresis (cDEP) and EK pumping, containing insulator obstacles for trapping and enrichment applications. They manipulated bioparticles at relatively lower applied electric potential using ACiDEP and EK pumping. AC electric field allows adjust- ing the dielectrophoretic response of particles by changing the frequency of the applied AC voltage [34-36]. The traditional insulator-based DEP requires higher applied electric potential than those based on ACiDEP [37]. Consequently, the main drawback of traditional iDEP is the joule heating increment inside the microchannel due to a relatively high applied voltage.

In this study, the effect of insulating posts' arrangement and geometry on the particle trapping efficiency of an iDEP system was investigated. Red blood cells were considered as particles in a microchannel containing circular, diamond, and square insulating posts. Unlike studies in the literature, the particles in this study are influenced by flow velocity and pDEP force. In order to compute the velocity of the flow and electric field, the finite element method was employed. By comparing the experimental data from the study of Demierre et al [38] with numerical results, the proposed numerical simulation was validated. Trapping capacity and the force ratio of the perpendicular to longitudinal components were introduced as the trapping criteria of particles. The optimum shape and arrangements of the insulating post were captured by probing the trapping capacity and force ratio for a range of geometrical parameters of insulators. Finally, particle trapping was investigated by the particle tracing method, and the particle trapping efficiency of systems with different posts was compared.

\section{Governing equation}

\subsection{DEP force}

DEP force is described as a force that is applied to a dielectric particle in a non-uniform electric field. We can define DEP force exerted on a spherical particle as follows [39]:

$$
F_{D E P}=2 \pi \varepsilon_{m} R^{3} \operatorname{Re}\left[f_{C M}\right] \nabla|E|^{2},
$$

where $\varepsilon_{m}$ is the permittivity of the medium, $R$ is the radius of the particle, $E$ represents the electric field, Re denotes the real part, and $f_{C M}$ is the Clausius-Mossotti factor, which can be stated as follows:

$$
f_{C M}=\frac{\varepsilon_{p}^{*}-\varepsilon_{m}^{*}}{\varepsilon_{p}^{*}+2 \varepsilon_{m}^{*}},
$$

where $\varepsilon_{p}^{*}$ and $\varepsilon_{m}^{*}$ correspond to the particle and medium complex permittivity, respectively. The complex permittivity is given as follows:

$$
\varepsilon^{*}=\varepsilon-j \frac{\sigma}{\omega},
$$

where $\varepsilon$ is the permittivity, $j$ is the imaginary unit, $\sigma$ is the conductivity, and $\omega$ is the angular frequency. Many of the biological cells can be described as a single shell. In order to estimate the permittivity of these particles, the following equation was used [29]: 


$$
\varepsilon_{p}^{*}=\varepsilon_{m e m}^{*} \frac{\left(\frac{R_{o}}{R_{i}}\right)^{3}+2\left(\frac{\varepsilon_{i}^{*}-\varepsilon_{m e m}^{*}}{\varepsilon_{i}^{*}+2 \varepsilon_{m e m}^{*}}\right)}{\left(\frac{R_{o}}{R_{i}}\right)^{3}-\left(\frac{\varepsilon_{i}^{*}-\varepsilon_{m e m}^{*}}{\varepsilon_{i}^{*}+2 \varepsilon_{m e m}^{*}}\right)},
$$

where $\varepsilon_{m e m}^{*}$ and $\varepsilon_{i}^{*}$ demonstrate the membrane and cytoplasm complex permittivity. Moreover, $R_{o}$ and $R_{i}$ are the outer and inner radii of the shell, respectively.

\subsection{Hydrodynamic friction (Stokes drag force)}

A particle in a flow is affected by the fluid velocity, which can be described by the hydrodynamic drag force. For a spherical particle, the hydrodynamic drag force is defined as follows $[29,36]$ :

$$
f_{\text {drag }}=6 \mu R \pi\left(u_{p}-u_{f}\right),
$$

where $u_{p}$ and $u_{f}$ are the velocity of the particle and medium, respectively, $\mu$ is the dynamic viscosity of the medium, and $R$ is the particle radius.

Figure 1(a) gives a schematic view of a particle in a microchannel with circular insulating posts along the flow streamlines. In addition, the two forces exerted on the particle are shown in Figure 1(b). The direction of DEP force and hydrodynamic drag force depends on the electric field and flow streamlines, respectively. The velocity, which is induced on the particle, can be computed by a balance between DEP force and hydrodynamic drag force $[29,36]$ :

$$
\vec{u}_{\mathrm{p}}=\vec{u}_{f}-\mu_{\text {dep }} \nabla E^{2}
$$

where $\mu_{d e p}$ is the dielectrophoretic mobility and can be calculated as follows:

$$
\mu_{d e p}=\frac{R^{2}}{3 \mu} \varepsilon_{m} \operatorname{Re}\left(f_{C M}\right) .
$$

Trapping of particles occurs when DEP force overcomes the hydrodynamic drag force in the flow direction. This can be stated as follows:

$$
\mu_{\text {dep }}=\frac{R^{2}}{3 \mu} \varepsilon_{m} \operatorname{Re}\left(f_{C M}\right) .
$$

Hence, the trapping criterion can be defined as follows:

$$
\frac{\mu_{d e p} \nabla E^{2}}{\vec{u}_{f} \cdot \vec{u}_{f}} \cdot \vec{u}_{f} \geq 1
$$

\section{Development of numerical simulation}

\subsection{Computational domain}

In this study, a microchannel containing arrays of distinctly shaped insulation posts is considered. Figure 2(a) presents one of the computational domains, which was employed in the current simulations. The width and length of the microchannel are constant and equal to 0.75 and $3.5 \mathrm{~mm}$, respectively, and the system contains just one inlet and one outlet for the fluid flow. Figure 2(b) depicts the configuration of three shaped insulation posts, considered in this study. In order to study the effects of the posts' arrangement on the particle trapping efficiency, three geometrical parameters were considered. $a / b$ represents the ratio of posts' length with respect to their width, $b_{s}$ is the perpendicular distance of the posts, and $a_{s}$ denotes their longitudinal distance.

\subsection{Computation of governing equations}

In this study, water was considered as the medium and, to configure particle specifications, the characteristics of red blood cells were implemented. Tables 1 and 2

Table 1. Medium characteristics.

\begin{tabular}{cccc}
\hline $\boldsymbol{\rho}_{\boldsymbol{m}}$ & $\boldsymbol{\mu}_{\boldsymbol{m}}$ & $\boldsymbol{\sigma}_{\boldsymbol{m}}$ & $\boldsymbol{\varepsilon}_{\boldsymbol{m}}$ \\
\hline $103 \mathrm{~kg} / \mathrm{m}^{3}$ & $1 \mathrm{mPa} . \mathrm{s}$ & $55 \mathrm{~ms} / \mathrm{m}$ & 80 \\
\hline
\end{tabular}

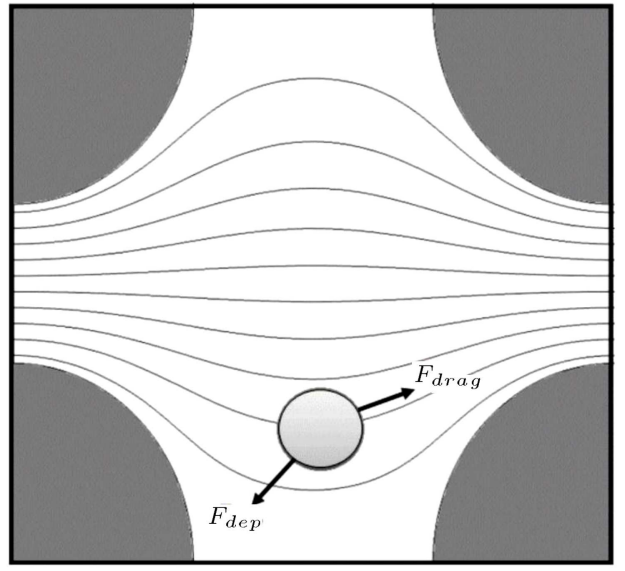

(a)

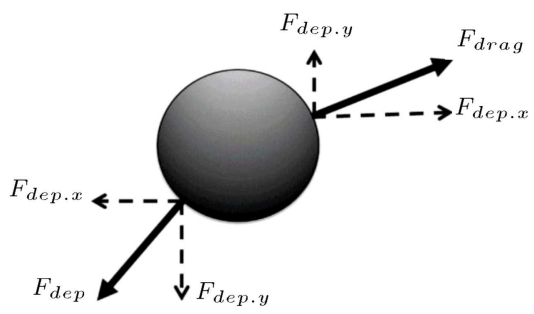

(b)

Figure 1. (a) Schematic diagram of the particle in a microchannel with circular insulating posts. (b) The forces exerted on the particle in the system. 


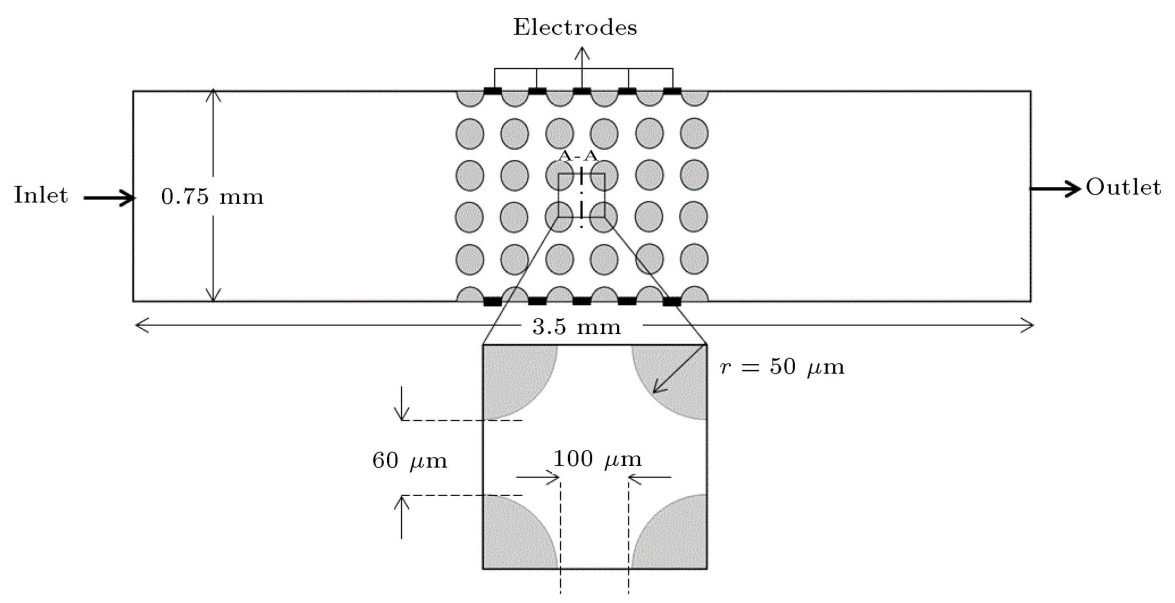

(a)
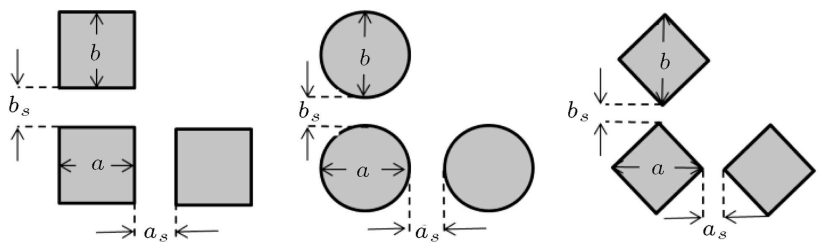

(b)

Figure 2. (a) Schematic representation of one of the computational domains. (b) Configuration of three shaped insulation posts besides three geometrical parameters considered in this study.

Table 2. Particle characteristics.

\begin{tabular}{ccccccc}
\hline $\boldsymbol{\rho}_{\boldsymbol{p}}$ & $\boldsymbol{r}_{\boldsymbol{o}}$ & $\boldsymbol{r}_{\boldsymbol{i}}$ & $\boldsymbol{\sigma}_{\boldsymbol{i}}$ & $\boldsymbol{\varepsilon}_{\boldsymbol{i}}$ & $\boldsymbol{\sigma}_{\boldsymbol{m e m}}$ & $\boldsymbol{\varepsilon}_{\boldsymbol{m e m}}$ \\
\hline $1050 \mathrm{~kg} / \mathrm{m}^{3}$ & $4 \mu \mathrm{m}$ & $3.91 \mu \mathrm{m}$ & $0.31 \mathrm{~s} / \mathrm{m}$ & 59 & $10^{-} 6 \mathrm{~s} / \mathrm{m}$ & 4.44 \\
\hline
\end{tabular}

present the medium and particle characteristics, which are employed in this study.

The inlet boundary condition was set as fully developed inflow with a peak velocity of $100 \mu \mathrm{m} / \mathrm{s}$. The ambient pressure was specified for the outlet boundary condition. The electrodes induced the electric field to the system with $V_{p p}=20 \mathrm{~V}$ and an angular frequency of $2 \mathrm{MHz}$. Considering medium and particle characteristics and angular frequency, the ClausiusMossotti factor is positive. Hence, the particles are influenced by positive dielectrophoretic force (pDEP). In order to calculate the governing equations, Finite Element Method (FEM) was used. For discretization of velocity and electric potential, quadratic interpolation and, for the pressure, linear interpolation were used.

\subsection{Particle tracing method}

Particle tracing method was used to compare the trapping efficiency of optimal insulating post shapes with other cases. Particle path can be determined based on the second law of Newton:

$$
F(t)=m \frac{d^{2} r}{d t^{2}}
$$

where $F$ is the combination of all forces exerted on the particle, $m$ is the particle mass, and $r$ is the particle position. An iterative approach was used to compute this Ordinary Differential Equation (ODE):

$$
r_{n+1}=0.5 \frac{F_{n}}{m} d t^{2}+v_{n} d t+r_{n}
$$

where $r_{n}$ and $r_{n+1}$ represent the position of the particle in previous and present time steps, $F_{n}$ denotes the combination of forces, and $v_{n}$ is the velocity of the particle that can be computed as follows:

$$
v_{n}=\frac{\left(r_{n}-r_{n-1}\right)}{d t}
$$

where $r_{n-1}$ is the position of the particle in two previous time steps. Combining Eqs. (11) and (12), we are able to describe the iterative method for particle tracing as follows [40]:

$$
r_{n+1}=2 r_{n}-r_{n-1}+0.5 \frac{F_{n}}{m} d t^{2} .
$$

In particle tracing simulation, the freeze boundary condition at walls is considered since the particles stick to the insulators in reality. 


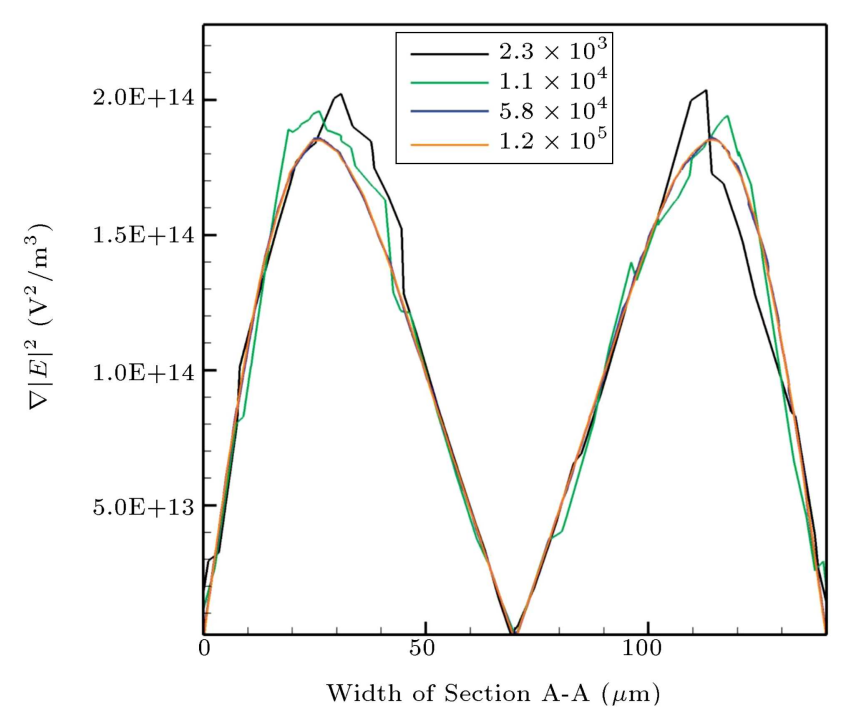

Figure 3. Independency of $\nabla|E|^{2}$ results with respect to the number of elements in Section A-A.

\subsection{Mesh generations}

For mesh generations, this study implemented triangular elements to enable complex geometry discretization. Due to the unstructured grid, grids with great flexibility around the posts were generated. For mesh independency studies, the $\nabla|E|^{2}$ profile in Section A-A (Figure 1(a)) was considered. As shown in Figure 3, the mesh refinement from $2.3 \times 10^{3}$ and $1.1 \times 10^{4}$ elements to $5.8 \times 10^{4}$ elements alters the $\nabla|E|^{2}$ profile. However, the use of a finer mesh with $1.2 \times 10^{5}$ elements does not change the velocity profile. Thus, the latter mesh is used for the computational results, presented in this paper.

\section{Results and discussion}

\subsection{Validation of numerical code}

In order to validate the FEM solver, the study of Demierre et al. [38] was employed. In this study, focusing of particles using DEP force was studied. The ratio of stream width of particles was measured with respect to channel width at the outlet for distinct flow velocities analytically and experimentally. Figure 4 depicts the computational domain of this study in

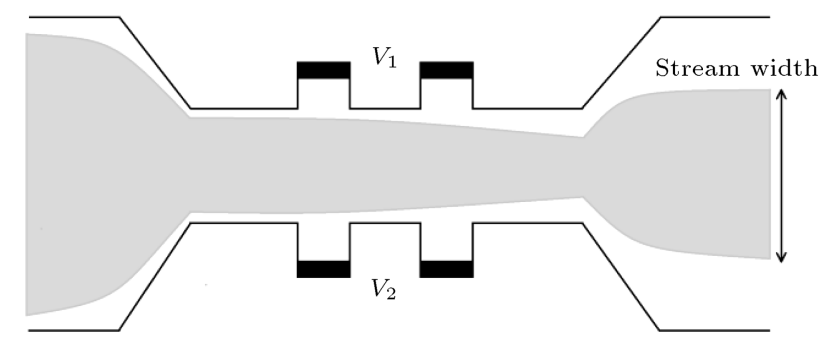

Figure 4. Schematic diagram of the computational domain for FEM solver validation. The highlighted section illustrates the particle's trajectory.

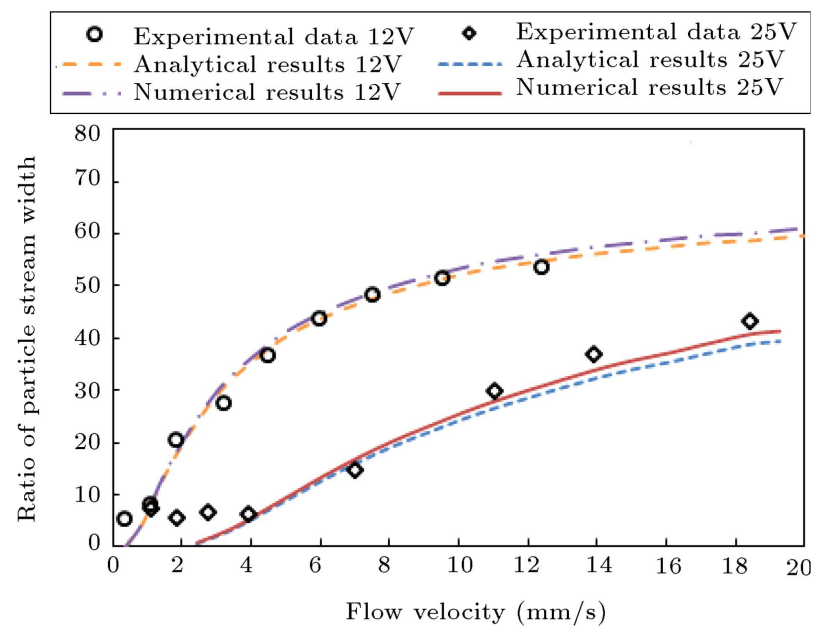

Figure 5. Schematic diagram of the computational domain for FEM solver validation.

addition to particles trajectory, which is illustrated by a highlighted section. Electric potentials $V_{1}$ and $V_{2}$ were applied identically with $\pi$ phase shift.

Figure 5 shows the comparison of numerical results and analytical and experimental data of Demierre et al. [35] in 2 distinct Electric potentials. Figure 5 shows good agreement between the FEM solver results and data of Demierre et al. [35], validating our numerical simulation.

\subsection{Velocity and $\nabla E^{2}$ distribution}

Velocity of the flow has significant effects on the trapping capacity of the particles. Figure 6 depicts the velocity of the flow distribution in the vicinity of distinct posts. The insulating posts in all three arrays share identical geometrical parameters. Diameter or width, length, and perpendicular and longitudinal distance of posts were set at 100, 100, 20, and 40 $\mu \mathrm{m}$, respectively. Since the cross-sectional area of the channel is the least between the posts, the maximum velocity occurs in this area in the three cases. The flow path around the square posts is more confined than that around the other two posts; hence, the velocity of the flow around the square post is higher than that around the circular and diamond posts. In addition, the area in which the maximum velocity occurs is further extended in the microchannel with square posts. In areas with low flow velocity, the time in which DEP force is exerted on the particles is longer since the particles take a longer time to cross the channel in these sections. Consequently, in channels with lower speed, the deviation of particles is the greatest.

The square of the electric field gradient $\left(\nabla E^{2}\right)$ distribution is considerably influenced by the shape of the posts. Figure 7 illustrates the distribution of $\nabla E^{2}$ around the circular, diamond, and square insulating posts. High magnitude of $\nabla E^{2}$ in a small area may 


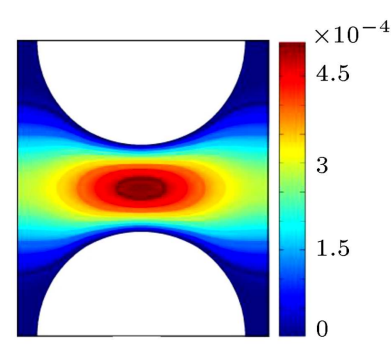

(a)

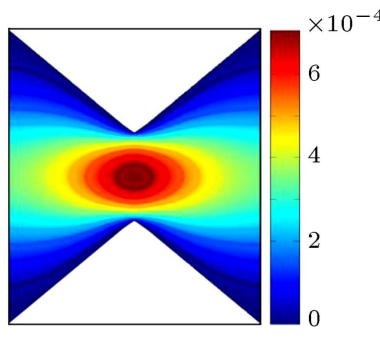

(b)

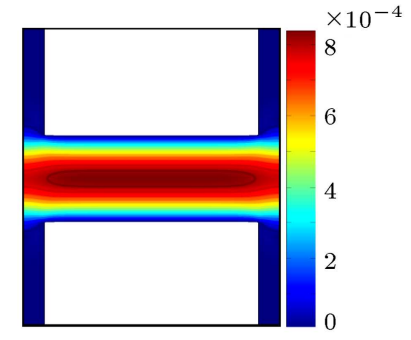

(c)

Figure 6. Velocity of the flow distribution in the microchannels with (a) circular, (b) diamond, and (c) square insulating posts.

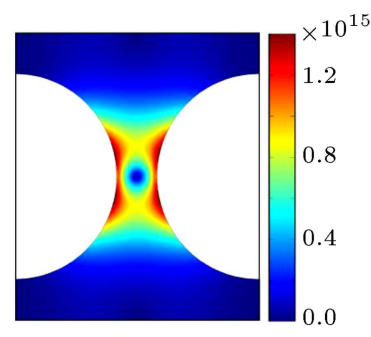

(a)

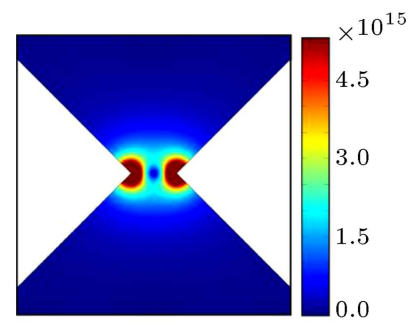

(b)

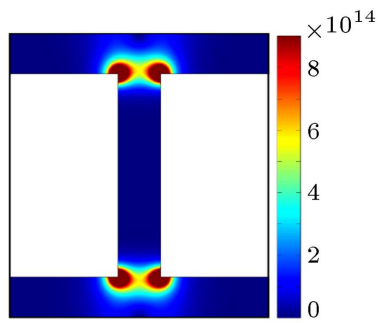

(c)

Figure 7. Distribution of $\nabla E^{2}$ in the microchannels with (a) circular, (b) diamond, and (c) square insulating posts.

not generate efficient particle movement. Thus, both of size and value of the area with a high magnitude of $\nabla E^{2}$ are important for study. The maximum $\nabla E^{2}$ occurs at angled points in microchannels with diamond and square posts, and the magnitude of $\nabla E^{2}$ alters instantly at the sharp and angled areas. However, the distribution of $\nabla E^{2}$ occurs even to a greater degree in the microchannel with circular posts. It should be noted that, in this study, all particles experience positive DEP. Hence, they were absorbed on areas with a high magnitude of $\nabla E^{2}$.

\subsection{Trapping capacities}

As discussed earlier, the combination of drag and DEP forces influences trapping efficiency. Drag and DEP forces are dependent on the velocity of the flow and electric field, respectively (considering Eqs. (1) and (5)). Further, the shape of the insulating posts affects the flow velocity and electric field. Thus, trapping efficiency can be modified by altering the posts' geometrical parameters. In order to investigate the effect of insulator shape on the trapping efficiency of the system, three geometrical parameters are considered: ratio of length to the width of posts $(a / b)$, perpendicular distance of the posts $\left(b_{s}\right)$, and longitudinal distance of the posts $\left(a_{s}\right)$.

Trapping efficiency of the system over a unit cell (as shown in Figure 2(a)) was assessed to compare the effect of geometrical parameters on the trapping efficiency. Hence, trapping capacity was defined as the average of dimensionless variables described in Eq. (9) over a unit cell:

$$
T_{C}=\frac{1}{A} \int\left|\frac{\mu_{d e p} \nabla E^{2}}{\vec{u}_{f} \cdot \vec{u}_{f}} \cdot \vec{u}_{f}\right| d A,
$$

where the area of the unit cell is denoted by $A$.

Trapping capacity can be employed to compare the effect of the insulating post's length and width $(a / b)$ on the particle trapping. However, $T_{C}$ is not the ideal criterion for examining the effect of perpendicular and longitudinal distances of the posts. Since DEP and drag force have the same direction and criterion in some cases by altering the distance of posts, $T_{C} \leq 1$ will not be satisfied, while particle trapping is observed. Thus, another concept was implemented to study the influence of post distances on trapping efficiency. The average force ratio $(F R)$ of the perpendicular to longitudinal components over a unit cell is described as follows:

$$
F R=\frac{1}{A} \int\left|\frac{F_{d r a g, y}+F_{d e p, y}}{F_{d r a g, x}+F_{d e p, x}}\right| d A,
$$

where $A$ denotes the area of the unit cell, and $F_{d e p}$ and $F_{\text {drag }}$ represent the DEP and drag force defined in Eqs. (1) and (5). The rate of particle transport towards the post can be determined by $F R$. For a system with larger $F R$, particles deviate quicker towards the post; therefore, the efficiency of particle trapping may be higher.

Figure 8 illustrates the percentage of average trapping capacity $\left(T_{C}\right)$ of the system with respect to $a / b$. It is noteworthy that the average trapping capacities are normalized using $T_{C}$ in $a / b=1$. This study probed the trapping capacity for $a / b=0.1$ to 6 


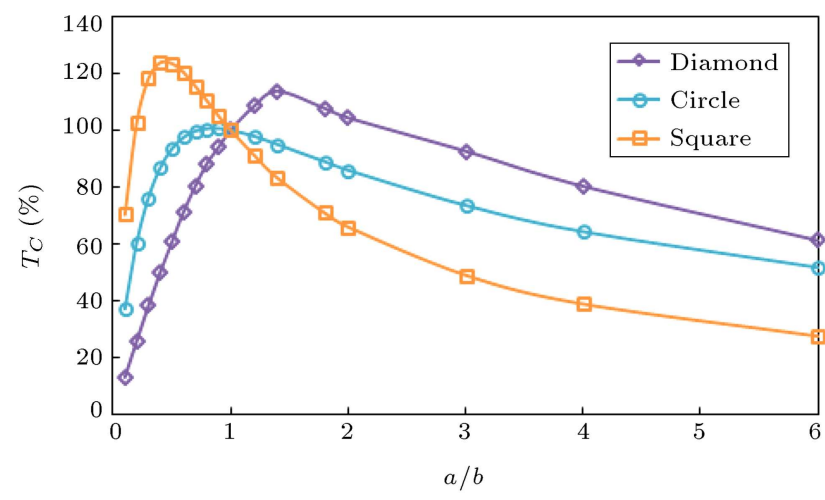

Figure 8. The percentage of trapping capacity $\left(T_{C}\right)$ with respect to the ratio of length to the width of posts $(a / b)$.

for microchannels with circular, diamond, and square insulating posts. Perpendicular and longitudinal distances of posts were set at 60 and $100 \mu \mathrm{m}$, respectively. For microchannels with square and circular posts, the most trapping capacity occurs in $a / b<1$ (narrowed posts). The highest trapping capacity of the system with square and circular posts is achieved in $a / b=0.5$ and $a / b=0.9$, respectively. However, in microchannels with diamond posts, the most trapping capacity was captured in $a / b>1$ (widened posts). The highest trapping capacity for the microchannel with a diamond post was observed in $a / b=1.4$. For the three posts, the trapping efficiency decreased when $a / b$ approached 0.1 and 6 . The variation of $T_{C}$ in square posts is more than that of diamond and circular posts, since the deviation of the electric field around the square post is sharper than that around the other two posts.

Figure 9 (a) presents the percentage of $F R$ with respect to the longitudinal distance of the posts $\left(a_{s}\right)$. The perpendicular distance of posts was set at $60 \mu \mathrm{m}$ and, for length and width of the posts, we employed the values in which the maximum trapping capacity was observed in the last figure. The width of posts (b) was fixed at $100 \mu \mathrm{m}$, and the length of the posts for square, circular, and diamond posts was set at 50, 90 , and $140 \mu \mathrm{m}$, respectively. The $F R$ of the system was calculated for $a_{s}=10$ to $400 \mu \mathrm{m}$. Similar to Figure 8 , the $F R$ was normalized using $F R$ value at $a_{s}=100 \mu \mathrm{m}$. In the three cases, the maximum $F R$ occurs in $a_{s} \leq 100 \mu \mathrm{m}$. The highest $F R$ for systems with square, circular, and diamond posts was observed in $a_{s}=50,40$, and $30 \mu \mathrm{m}$, respectively. Since the variation of $\nabla E^{2}$ is considerable at edge points of square and diamond posts (Figure $7(\mathrm{~b})$ and (c)), the variation of $F R$ in these two cases is more than that of circular posts. Similar to the last figure, $F R$ in all the cases decreased when $a_{s}$ approached 10 and $400 \mu \mathrm{m}$.

The percentage of $F R$ with respect to the perpendicular distance of the posts $\left(b_{s}\right)$ is illustrated in Figure $9(\mathrm{~b})$. Like the last case, the width of posts $(b)$ was fixed at $100 \mu \mathrm{m}$, and the length of the posts for

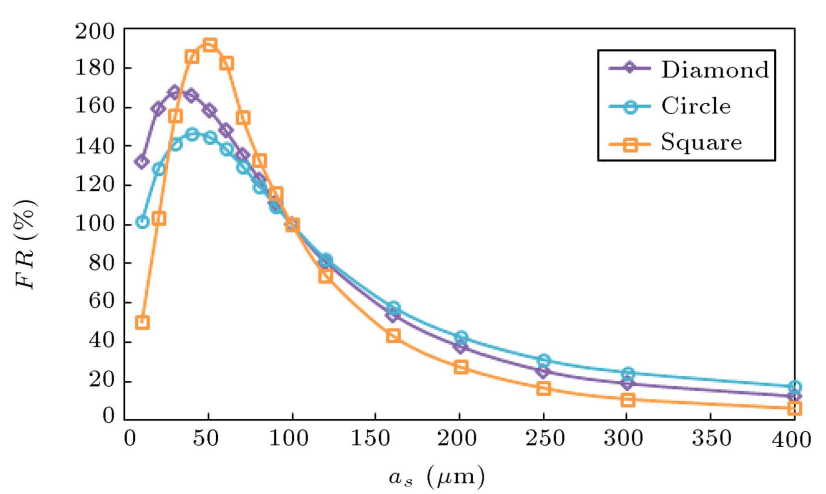

(a)

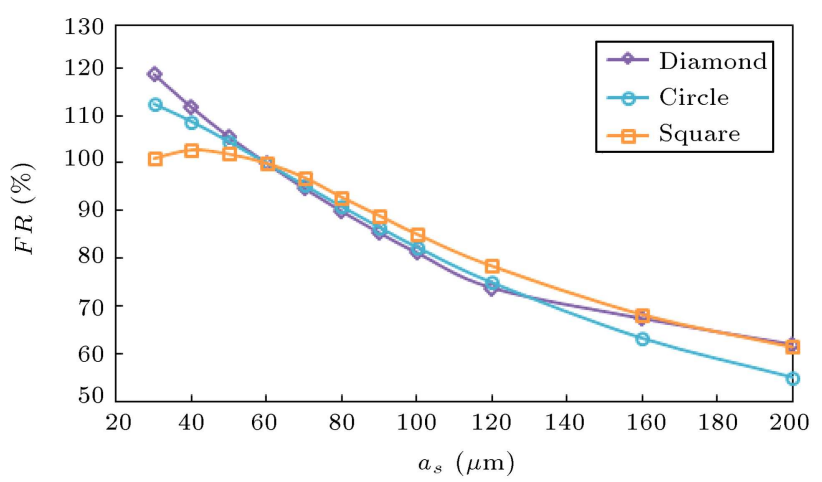

(b)

Figure 9. The percentage of $F R$ with respect to (a) longitudinal distance of the posts $\left(a_{s}\right)$ and $(\mathrm{b})$ perpendicular distance of the posts $\left(b_{s}\right)$.

square, circular, and diamond posts was set at 50, 90, and $140 \mu \mathrm{m}$, respectively. Further, the value of $a_{s}$ was extracted from the cases where the highest $F R$ was achieved in Figure 8. The longitudinal distance of the posts for square, circular, and diamond posts was determined to be 50,40 , and $30 \mu \mathrm{m}$, respectively. The $F R$ of the system was computed for $b_{s}=30$ to $200 \mu \mathrm{m}$. The value of $F R$ at $b_{s}=60 \mu \mathrm{m}$ was employed to normalize the $F R$. According to Figure 9(b), the maximum $F R$ for square, circular, and diamond posts occurred at $b_{s}=40,30$, and $30 \mu \mathrm{m}$, respectively. As shown in Figure 9(b), in the three cases, $F R$ decreased with an increase in the perpendicular distance of the posts.

\subsection{Particle tracing}

In order to observe the particle trapping, the particle tracing simulation was performed using Eq. (13). The particle trapping percentage of optimum shapes was compared with other cases to confirm the results of $T_{C}$ and $F R$. A hundred particles at the inlet of the microchannel were considered, and the number of immobilized particles between the insulating posts was computed to calculate the percentage of particle trapping.

Figure 10 presents the percentage of particle trapping efficiency for the three posts with distinct values of $a / b$ at $V_{p p}=25 \mathrm{~V}$. In the three posts, the optimum case 


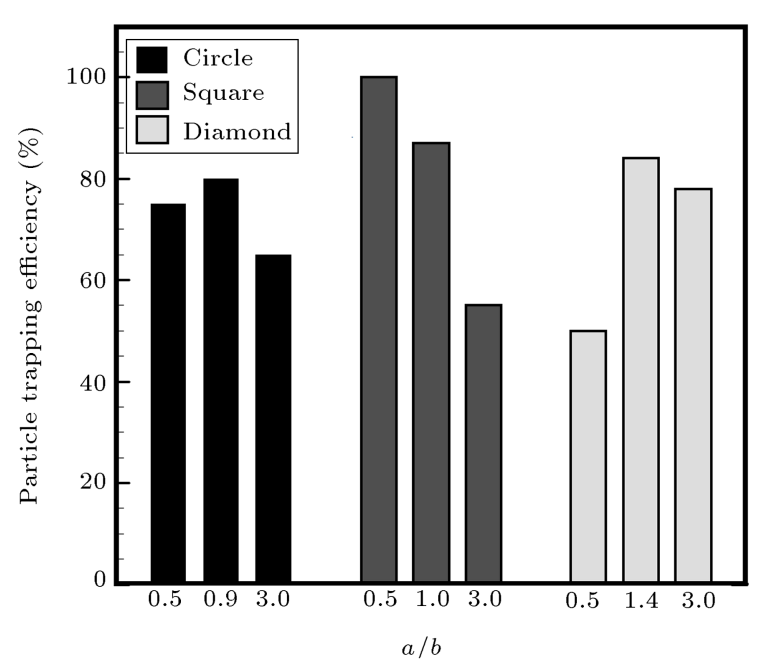

Figure 10. Particle trapping efficiency for microchannels with circular, diamond, and square insulating posts in district values of $a / b$ at $V_{p p}=25 \mathrm{~V}$.

of $a / b$ shows the highest percentage of particle trapping among other cases. In the microchannel with circular posts, the optimum value of $a / b$ is 0.9 , where particle trapping of $80 \%$ is achieved. In cases with narrower $(a / b=0.5)$ and wider posts $(a / b=3)$, the particle trapping is reduced to 75 and $65 \%$, respectively. The particle trapping of $100 \%$ was achieved in square posts with $a / b=0.5$, while an increase in the $a / b$ led to lower particle trapping efficiency. This is reasonable since $T_{C}$ is reduced with an increase in $a / b$. For microchannels with diamond posts, the particle trapping percentage of $84 \%$ at an optimum value of $a / b$ was computed. Reducing and increasing the $a / b$ leads to the lower particle trapping efficiency. It should be noted that the trapping efficiency in square posts is higher than that in other posts, since the maximum value of $\nabla E^{2}$ occurs relatively close to particle path lines in microchannels with square posts.

Figure 11 illustrates the particle trapping efficiency for different values of $a_{s}$ at $V_{p p}=20 \mathrm{~V}$. The maximum efficiency of systems with circular, square, and diamond posts reached 75,90 , and $100 \%$, respectively. Since the peak-to-peak electric potential is reduced with respect to the last figure, efficiency for the optimum case of circular posts reduces. However, microchannels with square and diamond posts showed a better performance despite the fact that $V_{p p}$ is reduced. In Figure 9(a), $F R$ is reduced with an increase in the optimal value of $a_{s}$. Consequently, the percentage of particle trapping reduces with an increase in the longitudinal distance of the posts. Similar to the last figure, systems with a square post showed higher trapping efficiency.

Particle trapping efficiency for distinct values of $b_{s}$ at $V_{p p}=18 \mathrm{~V}$ is depicted in Figure 12. As shown in Figure $9(\mathrm{~b})$, the value of $F R$ has an opposite relation

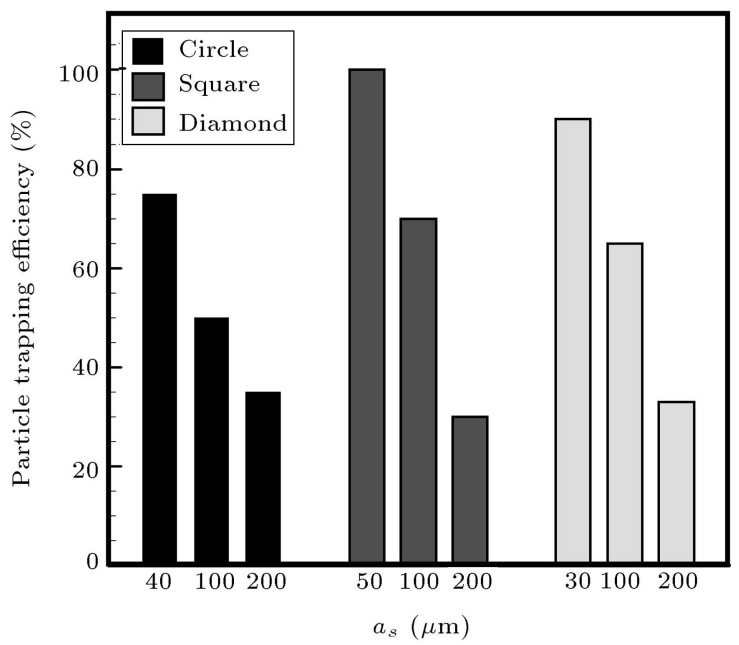

Figure 11. Particle trapping efficiency for microchannels with circular, diamond, and square insulating posts in district values of $a_{s}$ at $V_{p p}=20 \mathrm{~V}$.

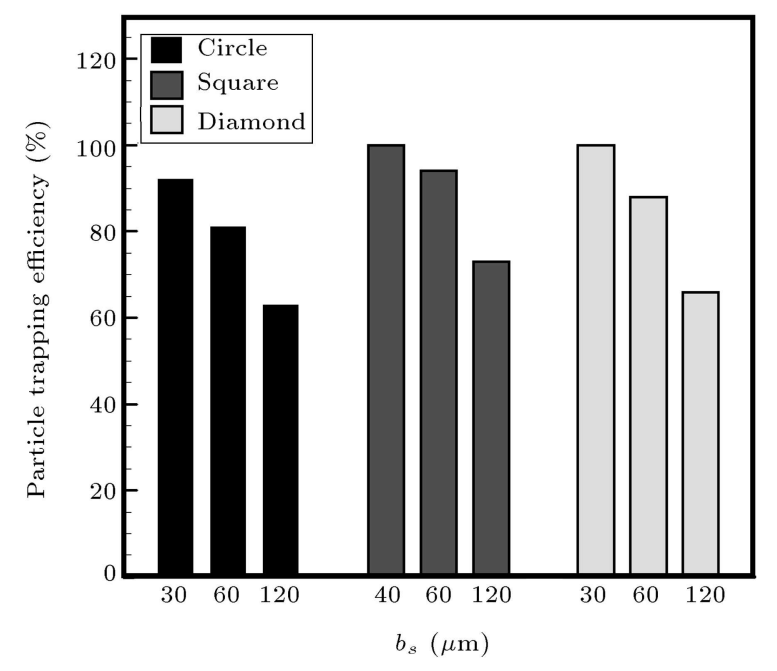

Figure 12. Particle trapping efficiency for microchannels with circular, diamond, and square insulating posts in district values of $b_{s}$ at $V_{p p}=18 \mathrm{~V}$.

with the perpendicular distance of the posts. Hence, a decrease in trapping efficiency with an increase in $b_{s}$ was observed. Although $V_{p p}$ was reduced, as shown in Figures 10 and 11, greater trapping efficiency was achieved at the optimal shape of all three cases.

\section{Conclusions}

The shape of the insulating post has significant effect on the velocity and electric field of iDEP systems. Since the trapping efficiency of an iDEP system is directly influenced by the velocity and electric field, the effect of arrangements and geometry of the insulating post on the trapping efficiency was chosen as the aim of this study. Systems with circular, square, and diamond insulators were considered to investigate the effect of 
their shape on the particle trapping performance. In order to calculate the velocity and electric field, the finite element method was employed, and the proposed numerical method was validated by comparing the results with experimental data of the study of Demierre et al. [38]. Perpendicular and longitudinal distances of the posts and ratio of width to length of insulators were selected as geometrical parameters to study their effects on the trapping efficiency. Trapping capacity $\left(T_{C}\right)$ and the force ratio $(F R)$ were introduced as the criteria for particle trapping. After assessing trapping capacity and $F R$ for distinct post arrangements and geometry, the optimum case for each system was captured. The highest trapping capacity of the system with square and circular posts was achieved in $a / b<1$ (narrowed posts), while the maximum trapping capacity occurred in $a / b>1$ in the diamond post case (widened posts). The highest $F R$ for systems with square, circular, and diamond posts was observed at longitudinal distances of 50,40 , and $30 \mu \mathrm{m}$, respectively, while the perpendicular distances of systems with square, circular, and diamond posts were 40,30 , and $30 \mu \mathrm{m}$, respectively, to achieve the highest $F R$. Finally, the particle tracing simulation was employed to show the particle trapping and confirm the results of trapping capacity and the force ratio. The highest particle trapping efficiency was observed in the case of narrower shapes for circular and square posts and wider shapes for diamond posts, as shown by trapping capacity results. Further, systems with square posts showed higher trapping performance than those with other posts.

\section{Nomenclature}

$\begin{array}{ll}E & \text { Electric field } \\ f_{C M} & \text { Clausius-Mossotti factor } \\ F R & \text { Average force ratio } \\ m & \text { Particle mass } \\ R & \text { Particle radius } \\ r & \text { Particle position } \\ T_{C} & \text { Trapping capacity } \\ u_{p} & \text { Particle velocity } \\ u_{f} & \text { Medium velocity } \\ \varepsilon_{p} & \text { Particle permittivity } \\ \varepsilon_{m} & \text { Medium Permittivity } \\ \mu & \text { Dynamic viscosity of the medium } \\ \sigma & \text { Conductivity } \\ \omega & \text { Angular frequency }\end{array}$

\section{References}

1. Çetin, B. and Li, D. "Dielectrophoresis in microfluidics technology", Electrophoresis, 32(18), pp. 2410-2427 (2011).
2. Gagnon, Z., Senapati, S., Gordon, J., et al. "Dielectrophoretic detection and quantification of hybridized DNA molecules on nano-genetic particles", Electrophoresis, 29(24), pp. 4808-4812 (2008).

3. Pethig, R. "Dielectrophoresis: Status of the theory, technology, applications", Biomicrofluidics, 4(2), pp. 510-713 (2010).

4. Whitesides, G. "The origins and the future of microfluidics", Nature, 4427101July, pp. 0028-0836 (2006).

5. Clark, I.C., Thakur, R., and Abate, A.R. "Concentric electrodes improve microfluidic droplet sorting", Lab on a Chip, 18(5), pp. 710-713 (2018).

6. Xuan, X., and Qian, S., Micro/Nano-Chip Electrokinetics, MDPI (2018).

7. Waheed, W., Alazzam, A., Mathew, B., et al. "Lateral fluid flow fractionation using dielectrophoresis (LFFFDEP) for size-independent, label-free isolation of circulating tumor cells", Journal of Chromatography B, 1087, pp. 133-137 (2018).

8. Flanagan, L.A., Lu, J., Wang, L., et al. "Unique dielectric properties distinguish stem cells and their differentiated progeny", Stem Cells, 26(3), pp. 656665 (2008).

9. Gagnon, Z.R. "Cellular dielectrophoresis: applications to the characterization, manipulation, separation and patterning of cells", Electrophoresis, 32(18), pp. 24662487 (2011).

10. Jones, T.B. and Jones, T.B., Electromechanics of Particles, Cambridge University Press (2005).

11. Choi, S., Ko, K., Lim, J., et al. "Non-linear cellular dielectrophoretic behavior characterization using dielectrophoretic tweezers-based force spectroscopy inside a microfluidic device", Sensors, 18(10), p. 3543 (2018).

12. Chen, X., Liang, Z., Li, D., et al. "Microfluidic dielectrophoresis device for trapping, counting and detecting Shewanella oneidensis at the cell level", Biosensors and Bioelectronics, 99, pp. 416-423 (2018).

13. Sato, N., Yao, J., Sugawara, M., et al. "Numerical study of particle-fluid flow under AC electrokinetics in electrode-multilayered microfluidic device", IEEE Transactions on Biomedical Engineering, 66(2), pp. 453-463 (2018).

14. Zhang, J., Yuan, D., Zhao, Q., et al. "Tunable particle separation in a hybrid dielectrophoresis (DEP)-inertial microfluidic device", Sensors and Actuators B: Chemical, 267, pp. 14-25 (2018).

15. Moncada-Hernández, H. and Lapizco-Encinas, B.H. "Simultaneous concentration and separation of microorganisms: insulator-based dielectrophoretic approach", Anal. Bioanal. Chem., 396(5), pp. 1805-1816 (2010).

16. Alazzam, A., Mathew, B., and Alhammadi, F. "Novel microfluidic device for the continuous separation of cancer cells using dielectrophoresis", J. Sep. Sci., 40(5), pp. 1193-1200 (2017). 
17. Cummings, E.B. and Singh, A.K. "Dielectrophoresis in microchips containing arrays of insulating posts: theoretical and experimental results", Anal. Chem., 75(18), pp. 4724-4731 (2003).

18. Lapizco-Encinas, B.H., Simmons, B.A., Cummings, E.B., et al. "Insulator-based dielectrophoresis for the selective concentration and separation of live bacteria in water", Electrophoresis, 25(10-11), pp. 1695-1704 (2004).

19. Aghaamoo, M., Aghilinejad, A., and Chen, X. "Numerical study of insulator-based dielectrophoresis method for circulating tumor cell separation, $M i$ crofluidics, BioMEMS, and Medical Microsystems XV, 10061, International Society for Optics and Photonics (2017).

20. Regtmeier, J., Käsewieter, J., Everwand, M., et al. "Continuous-flow separation nanoparticles by electrostatic sieving at micro-nanofluidic interface", J. Sep. Sci., 34(10), pp. 1180-1183 (2011).

21. Zellner, P., Shake, T., Sahari, A., et al. "Off-chip passivated-electrode, insulator-based dielectrophoresis (O-DEP)", Anal. Bioanal. Chem., 405(21), pp. 66576666 (2013).

22. Gallo-Villanueva, R.C., Pérez-González, V.H., Davalos, R.V., et al. "Separation of mixtures of particles in a multipart microdevice employing insulator-based dielectrophoresis", Electrophoresis, 32(18), pp. 24562465 (2011).

23. Moncada-Hernandez, H., Baylon-Cardiel, J.L., PérezGonzález, V.H., et al. "Insulator-based dielectrophoresis of microorganisms: Theoretical and experimental results", Electrophoresis, 32(18), pp. 2502-2511 (2011).

24. Kwon, J.-S., Maeng, J.-S., Chun, M.-S., et al. "Improvement of microchannel geometry subject to electrokinesis and dielectrophoresis numerical simulations", Microfluid. Nanofluid., 5(1), pp. 23-31 (2008).

25. Saucedo-Espinosa, M.A., and Lapizco-Encinas, B.H. "Experimental and theoretical study of dielectrophoretic particle trapping in arrays of insulating structures: Effect of particle size and shape", Electrophoresis, 36(9-10), pp. 1086-1097 (2015).

26. Pesch, G.R., Kiewidt, L., Du, F., et al. "Electrodeless dielectrophoresis: Impact of geometry and material on obstacle polarization", Electrophoresis, 37(2), pp. 291301 (2016).

27. LaLonde, A., Gencoglu, A., Romero-Creel, M.F., et al. "Effect of insulating posts geometry on particle manipulation in insulator based dielectrophoretic devices", $J$. Chromatogr. A, 1344, pp. 99-108 (2014).

28. Mohammadi, M., Zare, M.J., Madadi, H., et al. "A new approach to design an efficient micropost array for enhanced direct-current insulator-based dielectrophoretic trapping", Anal. Bioanal. Chem., 408(19), pp. 5285-5294 (2016).

29. Shafiee, H., Sano, M.B., Henslee, E.A., et al. "Selective isolation of live/dead cells using contactless dielectrophoresis (cDEP)", Lab Chip, 10(4), pp. 438-445 (2010).

30. Salmanzadeh, A., Romero, L., Shafiee, H., et al. "Isolatio of prostate tumor initiating cells through their dielectrophoretic signature", Lab Chip, 12(1), pp. 182-189 (2012).

31. Zellner, P., Shake, T., Hosseini, Y., et al. "3D Insulator-based dielectrophoresis using DC-biased, AC electric fields for selective bacterial trapping", Electrophoresis, 36(2), pp. 277-283 (2015).

32. Cristofanilli, M., Krishnamurthy, S., Das, C.M., et al. "Dielectric cell separation of fine needle aspirates from tumor xenografts", J. Sep. Sci., 31(21), pp. 3732-3739 (2008).

33. Sano, M.B., Gallo-Villanueva, R.C., Lapizco-Encinas, B.H., et al. "Simultaneous electro kinetic flow and dielectrophoretic trapping using perpendicular static and dynamic electric fields", Microfluid. Nanofluid., 15(5), pp. 599-609 (2013).

34. Martinez-Duarte, R., Camacho-Alanis, F., Renaud, P., et al. "Dielectrophoresis of lambda-DNA using 3D carbon electrodes", Electrophoresis, 34(7), pp. 11131122 (2013).

35. Demierre, N., Braschler, T., Muller, R., et al. "Focusing and continuous separation cells in micro fluidic device using lateral dielectrophoresis", Sensors and Actuators B: Chemical, $132(2)$, pp. 388-396 (2008).

36. Shamloo, A. and Kamali, A. "Numerical analysis of a dielectrophoresis field-flow fractionation device for the separation of multiple cell types", Journal of Separation Science, 40(20), pp. 4067-4075 (2017).

37. Saucedo-Espinosa, M.A., LaLonde, A., Gencoglu, A., et al. "Dielectrophoretic manipulation of particle mixtures employing asymmetric insulating posts", Electrophoresis, 37(2), pp. 282-290 (2016).

38. Demierre, N., Braschler, T., Linderholm, P., et al. "Characterization and optimization of liquid electrodes for lateral dielectrophoresis", Lab Chip, 7(3), pp. 355-365 (2007).

39. Çetin, B., Öner, S.D., and Baranoğlu, B. "Modeling of dielectrophoretic particle motion: Point particle versus finite-sized particle", Electrophoresis, 38(11), pp. $1407-1418$ (2017).

40. Mathew, B., Alazzam, A., Abutayeh, M., et al. "Model-based analysis of a dielectrophoretic microfluidic device for field-flow fractionation", J. Sep. Sci., 39(15), pp. 3028-3036 (2016).

\section{Biographies}

Reza Javidi received his MS degree in Mechanical 
Engineering from the University of Tehran, School of Engineering in 2017. His research interests include MEMS, computational mechanics, bio-MEMS, and nanotechnology.

Mahdi Moghimi Zand is an Assistant Professor at the School of Mechanical Engineering, College of Engineering, University of Tehran. He is also an Engineering and Research Consultant. Dr. Moghimi Zand's research is multi-disciplinary and revolves around vibrations, MEMS, computational mechanics, biomedical engineering, and nanotechnology.

Reza Nouri received his MS degree in Mechanical Engineering from the University of Tehran, School of Engineering in 2018. His research interests include electroosmotic and electrophoretic flows in the microchannel. 